\title{
A revision of Nearctic species of the genus Earota Mulsant \& Rey, 1874 (Coleoptera: Staphylinidae: Aleocharinae)
}

\author{
VLADIMIR I. GUSAROV \\ Division of Entomology, Natural History Museum, University of Kansas, Lawrence, KS 66045-7523, U.S.A. \\ and \\ Department of Entomology, St. Petersburg State University, Universitetskaya nab., 7/9, St. Petersburg 199034, \\ Russia \\ vlad@ku.edu
}

\begin{abstract}
Nearctic species of the genus Earota Mulsant \& Rey, 1874a are revised. Synonymy of generic names Earota and Macroterma Casey, 1906 is confirmed. Earota dentata (Bernhauer, 1906) (= E. alutacea $($ Casey, 1906) $=$ E. borealis $($ Casey, 1906) $=$ E. iowensis $($ Casey, 1910a) $)$ is recognized as the only valid Nearctic species of the genus. Redescription and illustrations are provided for distinguishing Earota from other aleocharine genera. Atheta sulcata Blatchley, 1910 is placed in synonymy with A. klimschi Bernhauer, 1909 and transferred from Earota to Atheta Thomson, 1858. Lectotypes for Atheta dentata Bernhauer, 1906, Macroterma alutacea Casey, 1906, M. borealis Casey, 1906, A. klimschi Bernhauer, 1909, A. iowensis Casey, 1910a and A. sulcata Blatchley, 1910 are designated.
\end{abstract}

Key words: Coleoptera, Staphylinidae, Aleocharinae, Athetini, Earota, Macroterma, taxonomy, Nearctic

\section{Introduction}

Mulsant and Rey (1874a) described the genus Earota to include the single species, Homalota reyi Kiesenwetter, 1850.

Bernhauer (1906) described Atheta dentata (now considered to be a member of Earota), and pointed out that this species represents "a distinct transition" between subgenera Aloconota Thomson, 1858, Liogluta Thomson, 1858 and Traumoecia Mulsant \& Rey, 1874a. He placed A. dentata in subgenus Liogluta, near A. (L.) monacha Bernhauer, 1899. 
Casey (1906) described the genus Macroterma to include two new species and later (Casey 1910a) added one additional new species.

Fenyes (1920) listed Macroterma as a synonym of Atheta (Earota), and placed the three species described by Casey in synonymy with $A$. (E.) dentata. This view was accepted by Bernhauer and Scheerpeltz (1926) but not by recent staphylinid workers (Seevers 1978; Newton et al. 2000). Bernhauer and Scheerpeltz (1926) included in Earota one additional Nearctic species (Atheta sulcata Blatchley, 1910).

In this paper I redescribe the genus Earota, revise the types of Nearctic species of Earota and reconfirm the synonymy established by Fenyes (1920). I follow the terminology accepted in taxonomy of Aleocharinae (Sawada 1970, 1972; Newton et al. 2000). A discussion of the terms applied to different parts of the internal sac of the aedeagus can be found in Gusarov (2002). To avoid the controversy on what side of the aedeagus should be called ventral (Gusarov 2002), I refer to the side of aedeagus bearing the basal orifice as parameral. The spermathecal gland is shown on the drawings solely to illustrate the gland position in relation to other parts of spermatheca.

The Fourth edition of the International Code of Zoological Nomenclature (ICZN 1999) requires (Article 74.7.3) a lectotype designation to "contain an express statement of the taxonomic purpose of the designation". The purpose of lectotype designations in this paper is to assure correct and consistent application of the names in the future. There is no reason to repeat this statement for each lectotype designation. All specimens designated as lectotypes were supplied with the red lectotype labels.

\section{Depositories}

AMNH American Museum of Natural History, New York (Dr. L.H.Herman)

CASC California Academy of Sciences, San Francisco (Dr. D.H.Kavanaugh)

CNCI Canadian National Collection of Insects, Ottawa (Mr. A.Davies)

FMNH Field Museum of Natural History, Chicago (Dr. A.F.Newton)

KSEM Snow Entomological Collection, University of Kansas (Dr. J.S.Ashe)

NMNH National Museum of Natural History, Washington, DC (Dr. T.L.Erwin)

PURC Purdue University, West Lafayette, Indiana (Dr. A.Provonsha)

SPSU Department of Entomology, St. Petersburg State University, St. Petersburg, Russia (Dr. V.I.Gusarov)

\section{Earota Mulsant \& Rey, 1874a (Figs. 1-34)}

Earota Mulsant \& Rey, 1874a: 154.

Earota Mulsant \& Rey, 1874b: 122 (synonymic homonym).

Macroterma Casey, 1906: 335 (as a genus in tribe Myrmedoniini Thomson, 1867). 
Atheta (Macroterma): Casey, 1910a: 9 (as valid subgenus).

Atheta (Macroterma): Casey, 1910b: 106 (as valid subgenus).

Atheta (Earota): Fenyes, 1920: 206 (as valid subgenus)

Atheta (Macroterma): Fenyes, 1920: 206 (as synonym of Atheta (Earota)).

Atheta (Earota): Bernhauer \& Scheerpeltz, 1926: 655 (as valid subgenus).

Atheta (Macroterma): Bernhauer \& Scheerpeltz, 1926: 655 (as synonym of Atheta (Earota)).

Ischnopoda (Earota): Blackwelder, 1952: 138 (as valid subgenus).

Ischnopoda (Macroterma): Blackwelder, 1952: 138 (as synonym of Ischnopoda (Earota)).

Earota: Seevers, 1978: 132 (as valid genus in subtribe Thamiaraeina Fenyes, 1921).

Macroterma: Seevers, 1978: 132 (as synonym of Earota).

Earota: Ashe in Newton, Thayer, Ashe \& Chandler, 2000: 371 (as valid genus; tribe Athetini

Casey, 1910a, not assigned to subtribe).

Macroterma: Ashe in Newton, Thayer, Ashe \& Chandler, 2000: 371 (as synonym of Earota).
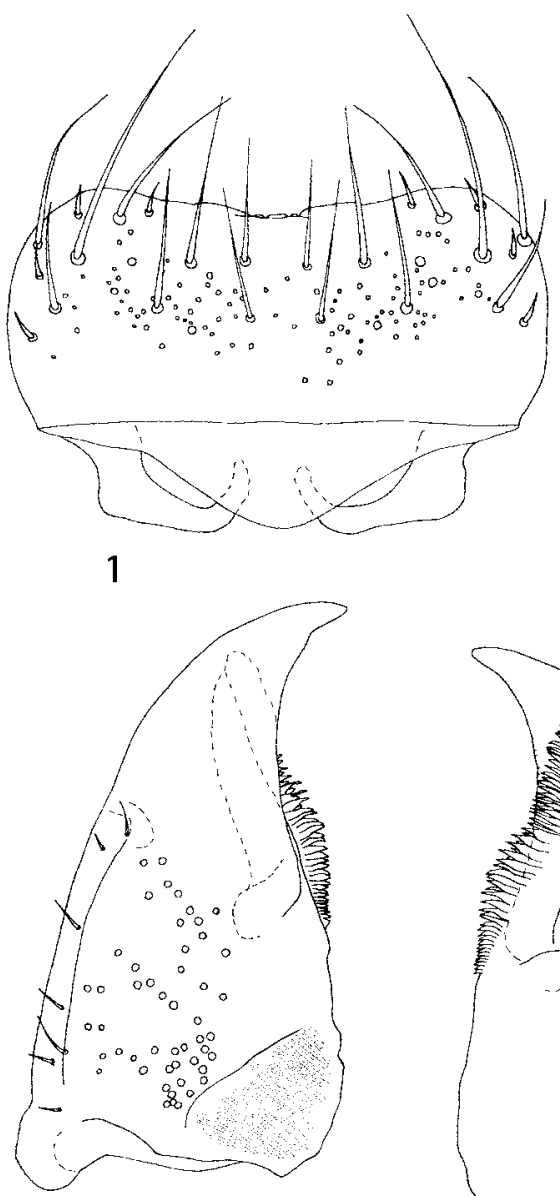

3
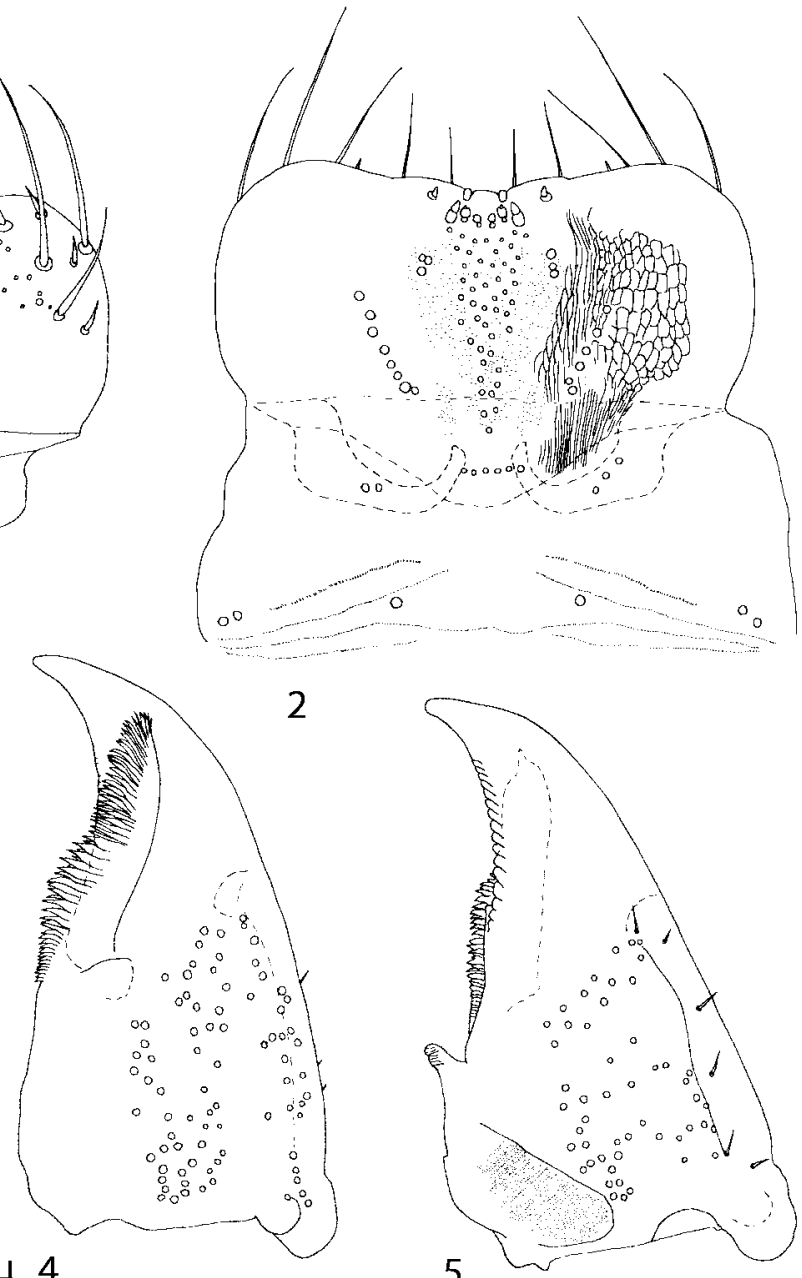

FIGURES 1-5. Mouthparts of Earota dentata (Bernhauer) (Terrace, British Columbia). 1 labrum; 2 - epipharynx; 3 - left mandible, dorsal view; 4 - left mandible, ventral view; 5 - right mandible, dorsal view. Scale bar $0.2 \mathrm{~mm}$. 
Diagnosis. Earota can be distinguished from other aleocharine genera by the combination of the following characters: broad body; last antennal article as long as three preceding combined; ligula with wide base, split into two lobes (Fig. 10); pronotum transverse, with microsetae directed posteriorly along the midline of the disc (Type II, Benick \& Lohse 1974) (Fig. 13); pronotal macrosetae moderately long; pronotal hypomera fully visible in lateral view; mesocoxae separated (Fig. 14); medial macroseta of mesotibia inconspicuous, shorter than tibial width; tarsal formula 4-5-5; metatarsal segment 1 slightly longer than segment 2; one empodial seta for each tarsus; apical process of median lobe of aedeagus straight or slightly bent ventrally in lateral view (Figs. 21, 26, 28).

Earota differs from Tropimenelytron Pace, 1983 by larger and broader body; base of ligula narrower; medial area of prementum with pseudopores; first segment of labial palpus with seta $\gamma$ present; complete infraorbital carina, transverse pronotum; and different shape of spermatheca.

Earota differs from Geostiba Thomson, 1858 by larger and broader body; first segment of labial palpus with seta $\gamma$ present; mesocoxae widely separated by broad mesosternal process; large and broad medial lamellae of internal sac of aedeagus; and different shape of spermatheca.

Earota differs from Pelioptera Kraatz, 1857 by larger body; large and broad medial lamellae of internal sac of aedeagus; and different shape of spermatheca.

Description. Length 3.0-4.5 mm. Body broad, dark brown with lighter elytra and legs.

Head as long as wide; eyes large, 1.2-1.5 times as long as temples; infraorbital carina complete. Antennal article 2 as long as article 3, article 10 transverse, article 11 as long as articles 8-10 combined (Fig. 6). Labrum (Fig. 1) transverse, anterior margin concave. Adoral surface of labrum (epipharynx) as in Fig. 2. Mandibles (Figs. 3-5) broad, medial tooth of right mandible inconspicuous; dorsal molar area with velvety patch consisting of tiny denticles (visible at 400x). Maxilla (Figs. 7-9) with galea extending slightly beyond apex of lacinia; apical lobe of galea covered with numerous fine and short setae; apex of lacinia with row of closely spaced spines, middle portion covered with numerous setae. Maxillary palpus with four segments (Fig. 7). Labium as in Figs. 10-12; labial palpi with three segments (Fig. 10); first segment with setae $\alpha$ and $\gamma$ present; ligula with wide base, split into two lobes; medial area of prementum with 9-12 pseudopores, lateral areas with 3 pores and single spinose pore. Hypopharyngeal lobes as in Fig. 11. Mentum (Fig. 12) with slightly concave anterior margin, medial area with numerous pores.

Pronotum (Fig. 13) transverse, broadest at middle, anterior margin straight, lateral and posterior margins convex; surface covered with microsetae directed posteriorly in midline, posteriorly and obliquely laterally in lateral areas (Type II, Benick \& Lohse 1974); macrosetae moderately long; hypomera fully visible in lateral view. Meso - metasternum as in Fig. 14, mesosternal process wide, extended about 1/2 length of mesocoxal cavities, metasternal process about $1 / 4$ length of mesocoxal cavities; mesosternum and mesosternal 
process not carinate medially; relative lengths of mesosternal process: isthmus: metasternal process in ratio of about 14:8:9; mesocoxal cavities margined posteriorly; mesocoxae separated. Medial macroseta of mesotibia inconspicuous, shorter than tibial width. Tarsal segmentation 4-5-5; metatarsal segment 1 slightly longer than segment 2 . One empodial seta. Wings fully developed. Posterior margin of elytra straight.

Abdominal terga 3-5 with moderately transverse basal impression. Tergum 7 as long as tergum 6. Punctation on terga 6-7 sparser than on terga 3-5. Tergum 7 with white palisade fringe.

Median lobe of aedeagus narrows apically (in parameral view; Figs. 20, 25, 27), apex straight or slightly bent ventrally (in lateral view; Figs. 21, 26, 28). Parameres with apical lobe bearing one long and three short macrosetae (Fig. 24). Copulatory piece of internal sac with narrow apical process (Figs. 29, 32-33). Medial lamellae (in ventral view) broad, apically with hook-like process each (Figs. 29, 31, 33).

Type species. Homalota reyi Kiesenwetter, 1850 by monotypy.

Discussion. The type species of Macroterma (M. alutacea, by original designation) shares with the type species of Earota all characters listed in the above description. The two species differ from each other only in proportions of antennal segments, in the shape of aedeagus and spermatheca, and in male secondary sexual characters of terga 7 and 8. Therefore, the commonly accepted synonymy of Earota and Macroterma (Fenyes 1920; Bernhauer \& Scheerpeltz 1926; Seevers 1978; Newton et al. 2000) is reconfirmed.

\section{Earota dentata (Bernhauer, 1906) (Figs. 1-32, 34)}

Atheta (Liogluta) dentata Bernhauer, 1906: 342 (publication date: September 30, 1906). Macroterma alutacea Casey, 1906: 335 (publication date: November 22, 1906). Macroterma borealis Casey, 1906: 336 (publication date: November 22, 1906). Atheta (Earota) dentata: Bernhauer, 1907: 393 (as valid species).

Macroterma borealis: Fenyes, 1907: 61 (as valid species).

Atheta dentata: Fenyes, 1908: 60 (as valid species).

Atheta alutacea: Fenyes, 1908: 61 (as valid species).

Atheta borealis: Fenyes, 1908: 61 (as valid species).

Atheta dentata: Blatchley, 1910: 354 (as valid species).

Atheta (Macroterma) dentata: Casey, 1910a: 9 (as valid species).

Atheta (Macroterma) alutacea: Casey, 1910a: 9 (as valid species).

Atheta (Macroterma) borealis: Casey, 1910a: 9 (as valid species).

Atheta (Macroterma) iowensis Casey, 1910a: 10.

Atheta (Macroterma) alutacea: Casey, 1910b: 106 (as valid species).

Atheta (Macroterma) dentata: Casey, 1910b: 106 (as valid species).

Atheta (Earota) dentata: Fenyes, 1920: 206 (as valid species).

Atheta (Earota) alutacea: Fenyes, 1920: 206 (as synonym of A. (E.) dentata).

Atheta (Earota) borealis: Fenyes, 1920: 206 (as synonym of A. (E.) dentata).

Atheta (Earota) iowensis: Fenyes, 1920: 206 (as synonym of A. (E.) dentata).

Atheta (Earota) dentata: Bernhauer \& Scheerpeltz, 1926: 655 (as valid species).

Atheta (Earota) alutacea: Bernhauer \& Scheerpeltz, 1926: 655 (as synonym of A. (E.) dentata). 

Atheta (Earota) iowensis: Bernhauer \& Scheerpeltz, 1926: 655 (as synonym of A. (E.) dentata). Earota dentata: Seevers, 1978: 271 (as valid species).

Earota alutacea: Seevers, 1978: 271 (as valid species).

Earota borealis: Seevers, 1978: 271 (as valid species).

Earota iowensis: Seevers, 1978: 271 (as valid species).

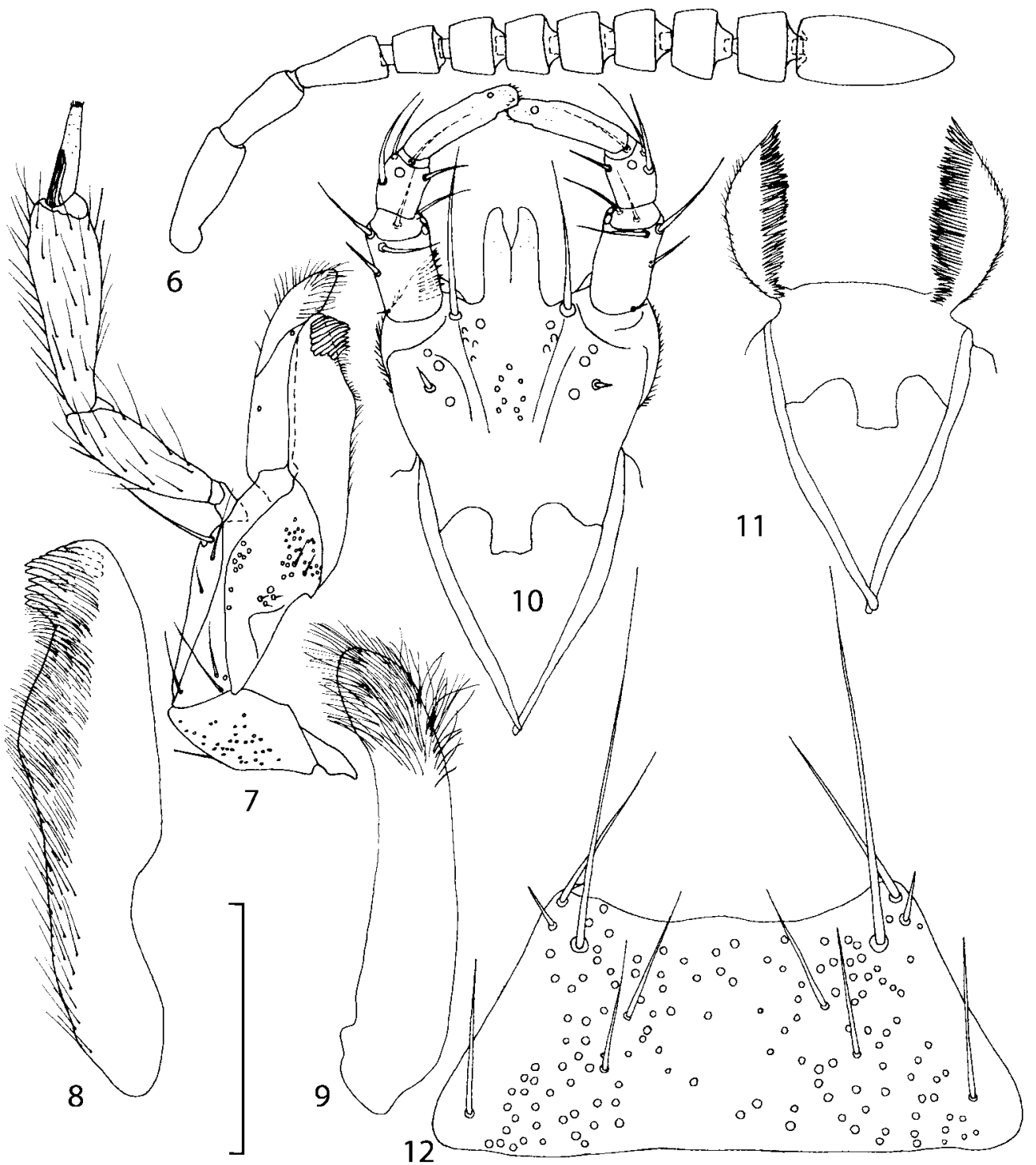

FIGURES 6-12. Mouthparts and antenna of Earota dentata (Bernhauer) (Terrace, British Columbia). 6 - antenna, 7 - right maxilla, ventral view; 8 - right lacinia, dorsal view; 9 - right galea, dorsal view; 10 - prementum; 11 - hypopharynx; 12 - mentum. Scale bar $0.4 \mathrm{~mm}$ (6), 0.2 $\mathrm{mm}(7), 0.1 \mathrm{~mm}(8-12)$. 
Type material. Lectotype of Atheta dentata (here designated), ox, "Ahwahnee Cal.",

"May", "dentata Brh. Cotypus" (yellow label), "Chicago NHMus M.Bernhauer Collection" (FMNH); 2 paralectotypes: o", "Wawona Cal.", "June", "dentata Bernh. Typus" (yellow label); 우, "Pasadena Cal.”, "Mar.”, “dentata Bh. Cotypus” (yellow label) (FMNH).

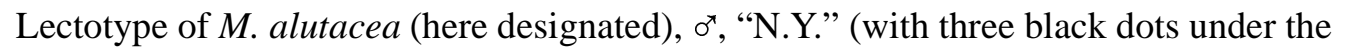
letters; Ithaca), "Macroterma alutacea Casey", "CASEY bequest 1925", "TYPE USNM 39454" (red label) (NMNH); 5 paralectotypes: $0^{x}, 4$ 우우, "N.Y." (with three black dots under the letters), "CASEY bequest 1925", "alutacea PARATYPE USNM 39454" (red label) (NMNH).

Lectotype of M. borealis (here designated), $\odot$, "Subalpine Mt. Wn [Washington]. N.H. vii.7.99", "borealis Csy.", "CASEY bequest 1925", "TYPE USNM 39455” (red label) (NMNH).

Lectotype of A. iowensis (here designated), ox, "Iowa City, Ia. Wickham.", "iowensis Csy.", "CASEY bequest 1925", "TYPE USNM 39456" (red label); paralectotype, 1 specimen with missing abdomen, "Iowa City, Ia. Wickham.", "CASEY bequest 1925", "iowensis-2 PARATYPE USNM 39456" (red label) (NMNH).

Additional material. CANADA: British Columbia: $390^{x} 0^{x}, 17$ 우우 $7 \mathrm{mi}$. E Terrace, river debris (Campbell \& Smetana), 26-27.vi.1968 (CNCI, KSEM, SPSU); o , $6.2 \mathrm{~km} \mathrm{~S}$ Terrace, $6 \mathrm{~km} \mathrm{E} \mathrm{Hwy.} \mathrm{25,} \mathrm{sifting} \mathrm{wet} \mathrm{moss} \mathrm{along} \mathrm{falls} \mathrm{of} \mathrm{small} \mathrm{fast} \mathrm{stream} \mathrm{(J.M.Campbell),}$ 25.viii.1983 (CNCI); ${ }^{\star}$, Shames River, $21 \mathrm{~km} \mathrm{~W}$ Terrace, 2 km N Hwy. 16, sifting alder and deciduous shrub litter (J.M.Campbell), 24.viii.1983 (SPSU); $20^{x} 0^{x}, 2$ 우 우, Creston (D.B.Waddell), 28.iv.1948; $\sigma^{x}$, ditto but 10.v.1948; $\sigma^{*}, 8 \mathrm{mi}$. W Creston, river debris (Campbell \& Smetana), 10.vi.1968; 우, 2 mi. S Salmo (Campbell \& Smetana), 9.vi.1968; ${ }^{x}$, Fort Nelson (M.T.Hughes), 26.viii.1948; ${ }^{x}$, Mt. Garibaldi, 14 mi. N Squamish, 4000' (Campbell \& Smetana), 30.v.1968; ox, Mt. Revelstoke N.P., Eva Lake, 6500' (J.M. \& B.A.Campbell), 25.vii.1971 (all - CNCI); 14 specimens, "Shngan Lake" (A.Fenyes) (CASC, KSEM, SPSU); Alberta: ${ }^{x}$, Seba, poplar woods (B.Hocking), 19.vii.1954 (CNCI); Manitoba: ㅇ, Winnipeg (J.B.Wallis), 24.v.1917 (CNCI); 2 specimens, ditto but no date (CASC); Québec: ${ }^{x}$, Saint-Jacques-de-Leeds, 46² $16^{\prime} \mathrm{N} 71^{\circ} 23^{\prime} \mathrm{W}$, maple forest,

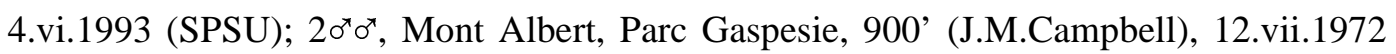
(CNCI); UNITED STATES: Maine: + , no locality (Frost) (CASC); New Hampshire: Carroll Co.: $0^{x}$, Intervale (A.Fenyes); Coos Co.: 우, Bretton Woods (A.Fenyes) (all CASC); county unknown: $\sigma^{x},+$, "Mt. Plst.Hse.", July (CASC, FMNH); Massachusetts: ㅇ, no locality (Blanch.); Middlesex Co.: $\sigma^{\star}$, Tyngs.[boro] (Bowditch) (all - CASC); 3 specimens, ditto but Merrimack River Drift, 25.iv.1901 (CASC, FMNH); $o^{x}$, Framingham (Frost); $\sigma^{x}$, S Framingham (Frost) (all - CASC); New Jersey: + , no locality, (NMNH (Casey collection)); Pennsylvania: ${ }^{x}$, no locality (Bang-Haas) (FMNH); Allegheny Co.: $\varsigma^{x}$, 2우오, Pittsburg (Klages), July (CASC, FMNH); Westmoreland Co.: 11 specimens, Jeannette (H.G.Klages) (CASC, FMNH, KSEM, SPSU); ${ }^{\star}$, ㅇ, ditto but July (CASC); 
Maryland: Baltimore Co.: ${ }^{x}$, Baltimore (F.E.Blaisdell), 10.v.1909; Montgomery Co.: ${ }^{x}$, ㅇ, Plummers I. (F.E.Blaisdell), 30.v.1909 (all - CASC); District of Columbia: $\sigma^{x}$, Washington (Schwarz), 22.ii.1886 (FMNH); Virginia: 우, no locality (NMNH (Casey collection)); North Carolina: 3 specimens, no locality (CASC, FMNH); Yancey Co: 우,

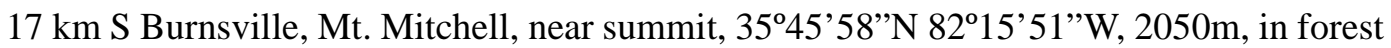
litter, Picea rubens, Abies fraseri (V.I.Gusarov), 3.vi.2001; Swain Co.: 우, $19 \mathrm{~km} \mathrm{NW}$ Cherokee, Clingmans Dome, S slope, 3533.66'N 83⒉9' W, 2000m, in forest litter, under young Abies fraseri (V.I.Gusarov), 1.vi.2001; Mitchell Co.: ㅇ, Blue Ridge Parkway, 9 km

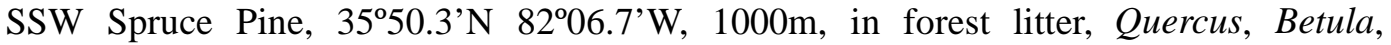
Rhododendron (V.I.Gusarov), 17.iii.2001 (all - SPSU); Buncombe Co.: 4 specimens, Asheville, 2.ii.1913 (CASC, FMNH); Alabama: Lee Co.: 30 $0^{x} 0^{x}$, 4우우, Auburn (E.J.Kiteley), 14.x.1982 (CNCI, SPSU); Ohio: $0^{\star}$, no locality (Hill) (CASC); Hamilton Co.: $0^{x}$, Cincinnati (NMNH (Casey collection)); 2 specimens, ditto but (Dury) (CASC);

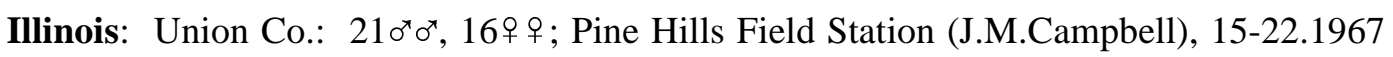

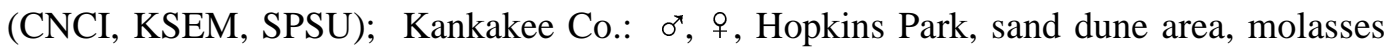
trap in burrow of Geomys b. illinoensis (H.S.Dybas \& R.L.Wenzel), 28.v.1946 (FMNH); Indiana: , no locality (NMNH (Casey collection)); Iowa: 2 우우, no locality (NMNH (Casey collection)); Kansas: Douglas Co.: 우, $1.5 \mathrm{~km} \mathrm{~N}$ Lawrence, right bank of Kansas

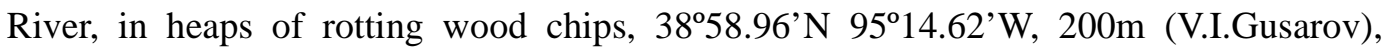
1.v.1999 (SPSU); Colorado: $0^{x}$, no locality (Klages) (CASC); Boulder Co.: $0^{x}, 2 \mathrm{mi} . \mathrm{W}$ Boulder, 5400' (J.M.Campbell), 8.viii.1973 (CNCI); Idaho: Latah Co.: (FMNH); Montana: Flathead Co.: 3 specimens, Kalispell (Wickham), June (CASC);

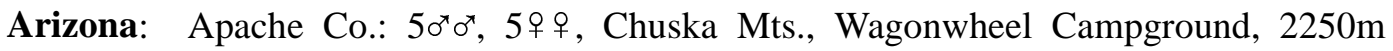
(J.M.Campbell), 12.vii.1976 (CNCI, KSEM, SPSU); Navajo Co.: $0^{x}$, ㅇ, 2 mi. W Black

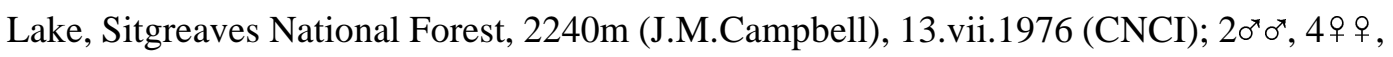
ditto but ex squirrel midden, 14.vii.1976 (CNCI, SPSU); Coconino Co.: 9 specimens, Flagstaff (A.Fenyes) (CASC, KSEM, SPSU); New Mexico: Otero Co.: $\sigma^{x}$, Lincoln National Forest, 2 mi. SE Cloudcroft, 8500' (A.Smetana), 13.vii.1969 (CNCI); $40^{\star} 0^{x}$, ditto but 1 mi. SE Cloudcroft, 8750', 13-18.vii.1969 (CNCI); ơ , ditto but 14.vii.1969 (CNCI); Lincoln Co.: 2우오, Sierra Blanca Ski Area, 10600' (A.Smetana), 13.vii.1969 (CNCI); $30^{x} 0^{x}$, 4 우우, ditto but 17.vii.1969 (CNCI, SPSU); San Miguel Co.: 5 specimens, El Porvenir (A.Fenyes) (CASC); Nevada: Lander Co.: $\sigma^{x}$, ㅇ, 17 mi. S Austin, Big Creek Canyon, $7400 \mathrm{ft}$ (L.Herman), 21.vi.1987 (AMNH); Alaska: ${ }^{x}$, Hess Creek, mi. 24 of Wales

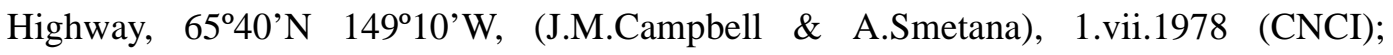
Washington: Pierce Co.: 우, Mt. Rainier National Park, N Puyallup River, 3700' (A. \& Z.

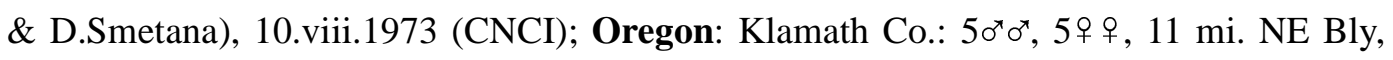
Deming Creek, 5000' (J.Schuh \& J.M.Campbell), 21.vii.1979 (CNCI, SPSU); Lake Co.: ${ }^{x}, 31.3$ mi. NW Lake View, Forest Roads $28 \& 3428 ; 5400 \mathrm{ft}$, spring runoff, alder litter near stream (L.Herman), 31.vii.1992 (AMNH); Baker Co.: 우, 12 mi. NW Unity, 3 mi. E of Forest Road 2675, W Fork Burnt River, 4600 ft (L.Herman), 28.vii.1992 (AMNH); Linn 
Co.: 우, Albany (Wickham), 15.vii.1923 (CASC); California: Los Angeles Co.: 34 specimens, Pasadena (A.Fenyes), January (CASC; FMNH, KSEM, SPSU); 3 specimens, ditto but December (CASC); + , ditto but iv.1906; 7 specimens, ditto but no date; 3 specimens, Mt. Wilson (A.Fenyes); 4 specimens, mountains near Claremont (Baker); 우, Redondo, April (all - CASC); Marin Co.: 6 specimens Glenn Ranch (CASC, FMNH); Mariposa Co.: 1 specimen, Summerdale (A.Fenyes) (CASC); Mono Co.: ${ }^{x}, 6 \mathrm{mi} . \mathrm{SW}$ Toms Place, 9000' (A.Smetana), 8.viii.1969 (CNCI); Placer Co.: 3 specimens, Applegate (A.Fenyes) (CASC); San Bernardino Co.: 우, San Bernardino Mts, 1 mi. E Fallsvale, 6200' (A.Smetana), 10.iii.1983 (CNCI); 6 specimens, San Bernardino Mts. (CASC); San Diego Co.: ơ, Mt. Palomar, 5000' (J.M.Campbell), 27.xi.1981 (CNCI); + , Oceanside (A.Fenyes); San Francisco Co.: ${ }^{\star}$, (Blaisdell), July; Santa Clara Co.: 5 specimens, Los Gatos (A.Fenyes); Sonoma Co.: $0^{x}$, Occidental, 4.vi.1920 (all - CASC); county unknown: 3 specimens, Miami, June (CASC, FMNH); 2 specimens, Sugar Pine (A.Fenyes) (CASC).

Diagnosis. Earota dentata can be distinguished from the West Palaearctic E. reyi by having subquadrate antennal article 4, more transverse articles 5-10, more narrow median lobe of aedeagus (in parameral view), more narrow medial tubercle on male tergum 7 and protruding apical lobe on male tergum 8.
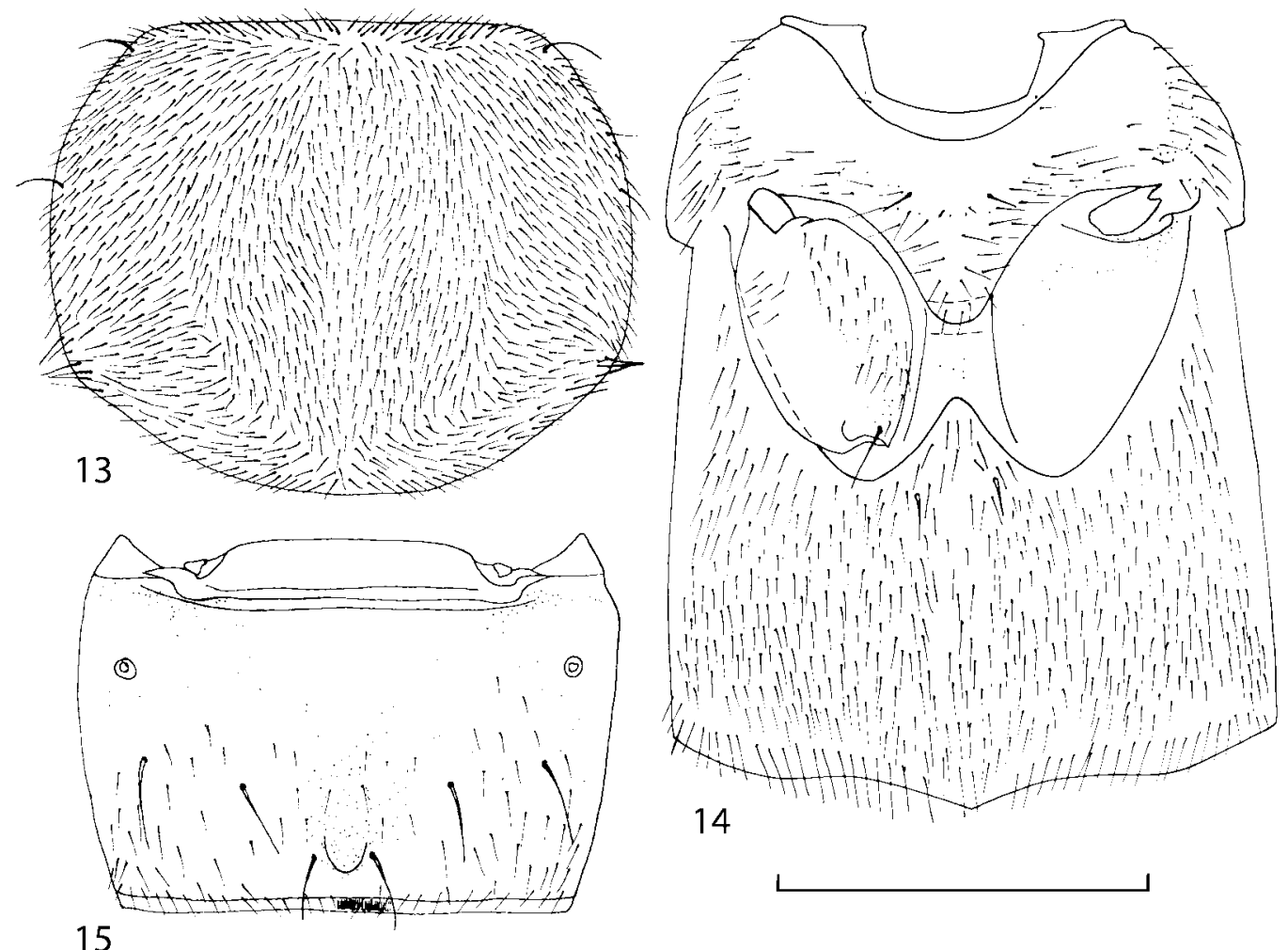

FIGURES 13-15. Details of Earota dentata (Bernhauer) (Terrace, British Columbia). 13 pronotum; 14 - mesometathorax, ventral view; 15 - male tergum 7 . Scale bar $0.5 \mathrm{~mm}$. 
Description. Length 3.0-4.5 mm. Dark brown, elytra and legs lighter, reddish brown to brownish yellow.

Head subquadrate, surface glossy, on disk with weak isodiametric microsculpture, punctation fine, distance between punctures equals 2-3 times their diameter. Eyes 1.2-1.5 times as long as temples (seen from above). Second antennal article as long as third, article 4 subquadrate, articles 5-10 transverse (width to length ratio 1.3-1.6), last article as long as three preceding combined (Fig. 6).

Pronotum transverse, 1.2-1.3 times as wide as head, width to length ratio 1.2-1.3, broadest at middle, anterior margin straight, lateral and posterior margins convex; surface glossy, punctation and microsculpture as on head. Elytra 1.2-1.4 times as wide and 1.3-1.4 times as long (measured from humeral angle) as pronotum, 1.2-1.3 times as wide as long, glossy, microsculpture and punctation as on head, punctures slightly asperate.

Abdominal terga glossy, with fine punctation and microsculpture. Terga 3-6 with microsculpture consisting of transverse waves, tergum 7 with microsculpture of transverse waves or strongly transverse meshes. On terga 3-5 distance between punctures equals 2-5 times their diameter, on terga 6-7 distance between punctures equals 3-6 times their diameter, in some specimens tergum 7 impunctate along midline.

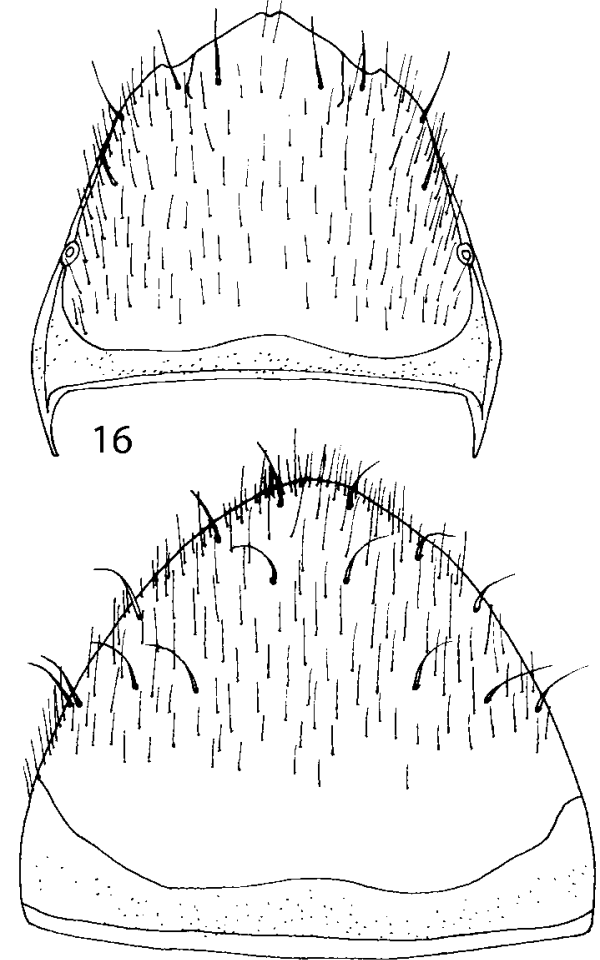

17

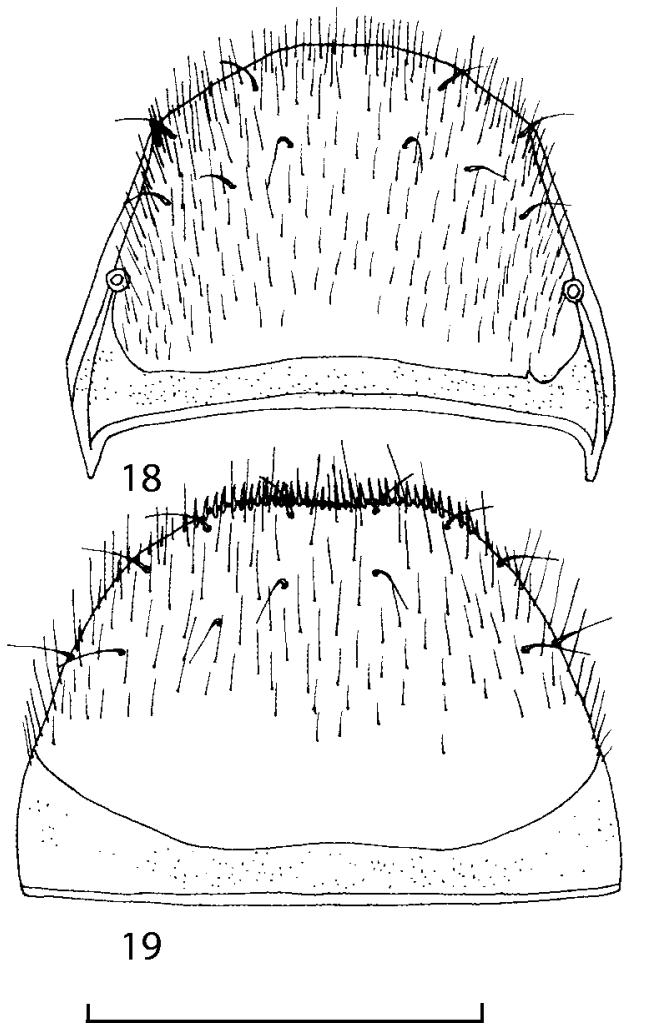

FIGURES 16-19. Details of abdominal segment 8 of Earota dentata (Bernhauer) $(16,18-19$, Terrace, British Columbia; 17, Black Lake, Arizona). 16 - male tergum 8; 17 - male sternum 8; 18 - female tergum $8 ; 19$ - female sternum 8 . Scale bar $0.5 \mathrm{~mm}$. 
Male tergum 7 with short medial carina in front of anterior margin (Fig.15). Male tergum 8 with protruding lobe, which is emarginate apically (Fig. 16). Posterior margin of male sternum 8 convex (Fig. 17). Aedeagus as in Figs. 20-21, 24-32.

Female tergum 8 with convex posterior margin (Fig. 18). Sternum 8 with slightly concave posterior margin (Fig. 19). Spermatheca as in Figs. 22-23.

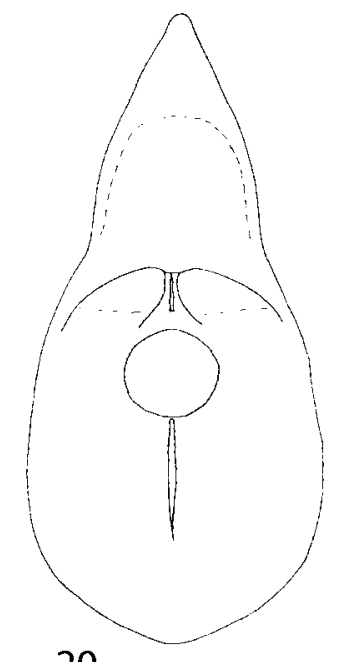

20

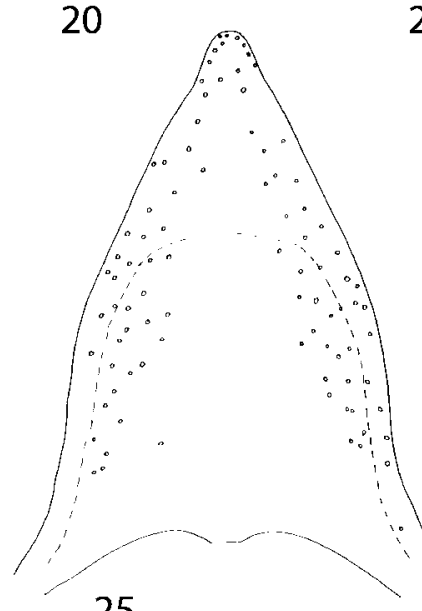

25

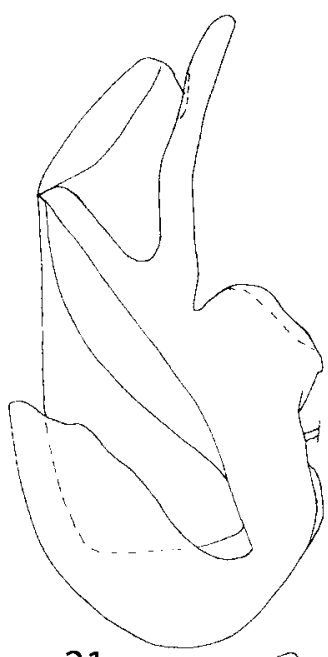

21
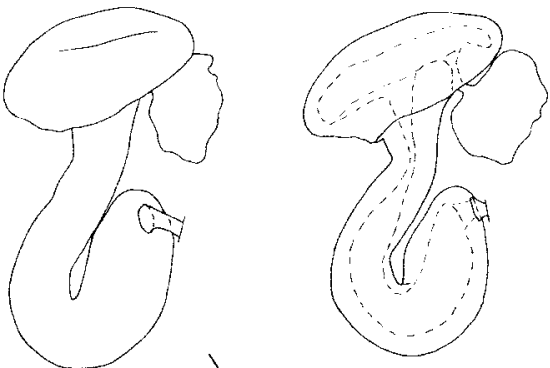

22 23

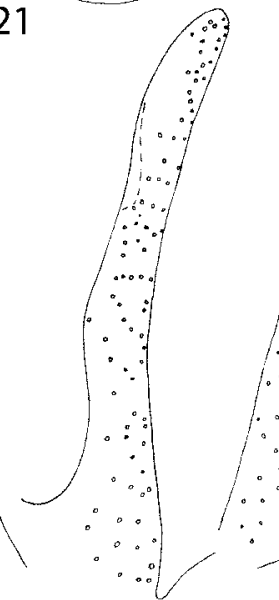

26

\section{7}

28

FIGURES 20-28. Genitalia of Earota dentata (Bernhauer) (20-21, 25-26, Black Lake, Arizona; 22-24, Terrace, British Columbia; 27-28, Pine Hills Station, Illinois). 20 - median lobe of aedeagus, parameral view; 21 - median lobe of aedeagus, lateral view; 22-23 - spermatheca; 24 apical lobe of left paramere, side facing median lobe; 25, 27 - apex of median lobe, parameral view; 26, 28 - apex of median lobe, lateral view. Scale bar $0.4 \mathrm{~mm}$ (20-23), $0.2 \mathrm{~mm}$ (24-28).

Discussion. Casey (1910b) believed that E. dentata, described by Bernhauer (1906) from California, can be distinguished from E. alutacea by smaller and more narrow body. 
However, both small and large specimens are found in the same samples in different parts of North America and therefore the size alone cannot be used to assign the population from California to a separate species. The types of $E$. dentata and other examined specimens from California are similar to the specimens from other regions of North America in both external characters and genitalia.

The types of the three species described by Casey $(1906,1910 a)$ are similar to the types of $E$. dentata in both external characters and genitalia and I agree with the synonymy established by Fenyes (1920) and accepted by Bernhauer and Scheerpeltz (1926).
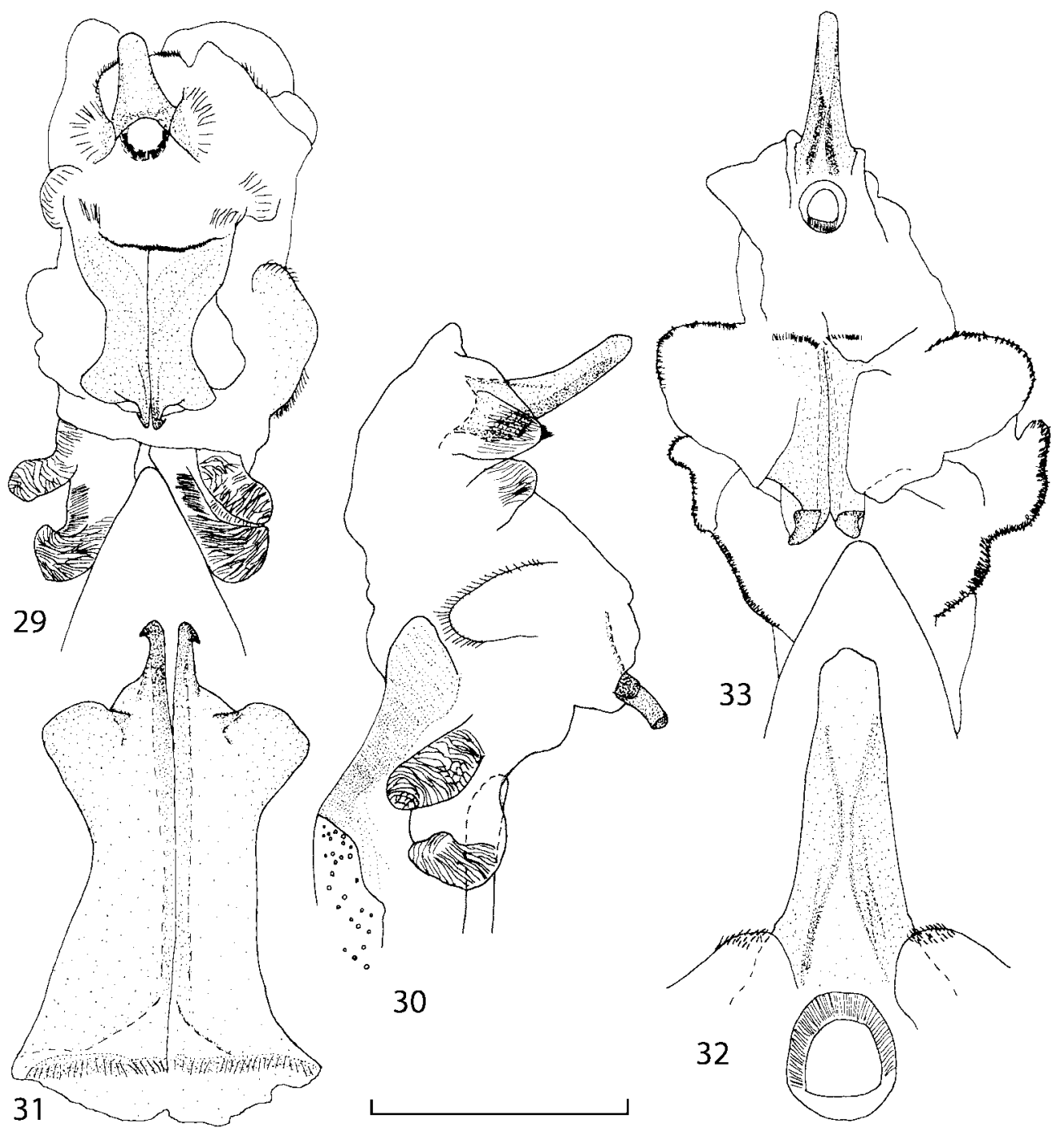

FIGURES 29-33. Male genitalia of Earota dentata (Bernhauer) (29-32, Terrace, British Columbia; 33, Mt. Pagano, Italy) and E. reyi (Kiesenwetter). 29, 33 - everted internal sac of aedeagus, parameral view; 30 - everted internal sac of aedeagus, lateral view; 31 - medial lamellae, ventral view; 32 - copulatory piece, ventral view. Scale bar $0.2 \mathrm{~mm}$ (29-30, 33), $0.1 \mathrm{~mm}$ (31-32). 
The shape of the apex of median lobe of aedeagus varies slightly (Figs. 25-28) but no

gaps or geographical clines were detected in examined material. This makes $E$. dentata the only valid species of Earota known in North America.

Distribution. Widespread in Canada and United States (Fig. 34).

Natural History. The specimens of E. dentata were collected in leaf litter, in moss, in river debris and in burrows of Geomys.

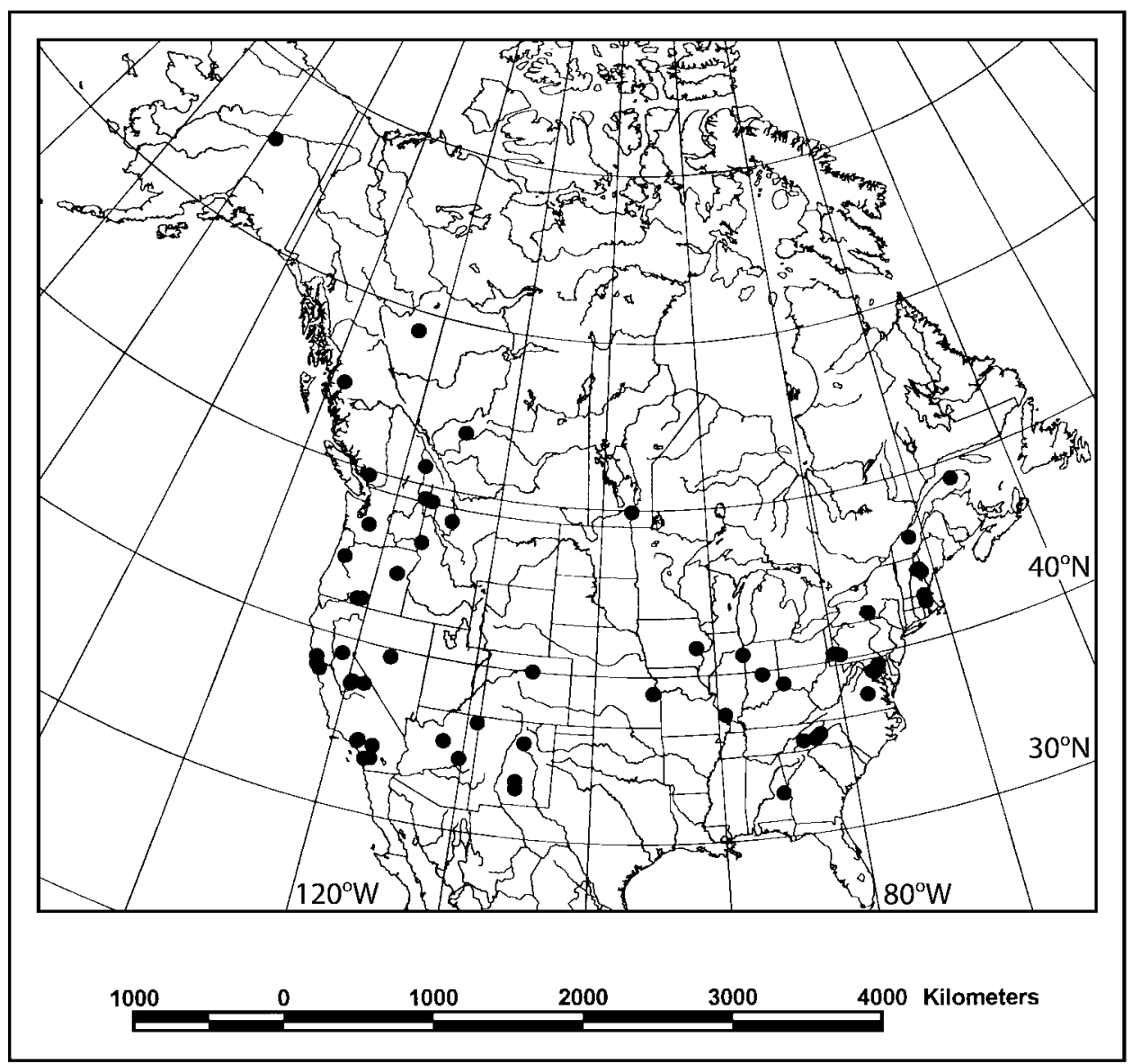

FIGURE 34. Geographical distribution of Earota dentata (Bernhauer) (•).

\section{Earota reyi (Kiesenwetter, 1850) (Fig. 33)}

Homalota reyi Kiesenwetter, 1850: 218.

Myrmedonia triangulifera Fairmaire, 1883: xli.

Earota reyi: Mulsant \& Rey, 1874a: 124.

(other references are omitted) 
Material examined. Italy: $\sigma^{x}$, + , Prov. di Aquila, Mt. Pagano (Paganetti) (FMNH).

Diagnosis. Earota reyi can be distinguished from the Nearctic E. dentata by having elongate antennal article 4, less transverse articles 5-10, broader median lobe of aedeagus (in parameral view), broad and flat triangular tubercle on male tergum 7 , straight posterior margin of male tergum 8 .

\section{Atheta klimschi (Bernhauer, 1909)}

Atheta (s. str.) klimschi Bernhauer, 1909: 523 (publication date: July 1, 1909).

Atheta sulcata Blatchley, 1910: 355 (publication date: September 20, 1910), syn. nov.

Atheta (Hypatheta) klimschi: Bernhauer \& Scheerpeltz, 1926: 653 (as valid species).

Atheta (Earota) sulcata: Bernhauer \& Scheerpeltz, 1926: 655 (as valid species).

Stethusa klimschi: Seevers, 1978: 267 (as valid species).

Earota sulcata: Seevers, 1978: 271 (as valid species).

Type material. Lectotype of Atheta klimschi (here designated), ox, "Opelousas, La. June", "Klimschi Brh. Typus. Klimsch" (yellow label), "Earota klimschi Brnh. Typ. Klimsch. det. Bernh.", "Chicago NHMus M.Bernhauer Collection" (FMNH); paralectotype, ơ, "Opelousas, La. June", "Klages", "Klimschi Brh. Cotypus. Klimsch" (yellow label), "Chicago NHMus M.Bernhauer Collection" (FMNH).

Lectotype of Atheta sulcata (here designated), ox, "TYPE" (red label), "Posey Co. Ind. W. S.B. 4-22-09”, “3618 det. A.Fenyes", "11”, "Purdue Blatchley collection”, "Atheta sulcata sp. nov." (PURC).

Additional material. ox, "Opelousas, La. May", "Klimschi Bernh. Cotypus. Klimsch" (yellow label), "Chicago NHMus M.Bernhauer Collection" (FMNH); ox, "Opelousas, La. May”, "als pedicularis von Klimsch”, "Klimschi Brh. Cotypus. Klimsch” (yellow label), "Chicago NHMus M.Bernhauer Collection" (FMNH).

Discussion. The two males listed as additional material were labeled by Bernhauer as cotypes. In his description of A. klimschi Bernhauer indicated that the specimens were collected in June. Therefore, the males collected in May had not been included in the type series of A. klimschi and cannot be considered the paralectotypes.

The types of A. klimschi and A. sulcata are identical in external characters and male genitalia. Although A. sulcata was included in Earota by Bernhauer and Scheerpeltz (1926) and Seevers (1978), this species has narrow mesosternal process, characteristic of Atheta, unlike both $E$. reyi and $E$. dentata which have mesocoxae widely separated by broad mesosternal process. Therefore, A. klimschi (=A. sulcata) cannot be included in the genus Earota and this species is considered here to be a member of Atheta. A detailed redescription of $A$. klimschi will be given in a separate paper. 
I am greatly indebted to Aleš Smetana, Anthony Davies, Lee Herman, Alfred Newton, Philip Parrillo, David Kavanaugh, Roberta Brett, Arwin Provonsha, Terry Erwin and Dave Furth for the loan of specimens deposited in their respective institutions. I am grateful to two anonymous referees for their comments which helped to improve my manuscript. I thank Margaret Thayer, Aleš Smetana and Derek Lott for discussing with me some terminology problems in Staphylinidae. Alexandria Digital Library Gazetteer Server (http://fat-albert.alexandria.ucsb.edu:8827/gazetteer/) was used to find coordinates for some localities. This work was supported by National Science Foundation PEET grants DEB-9521755 and DEB-9978110 to Steve Ashe and by the Russian Federal program "Russian Universities - Fundamental Sciences" (project 07.01.056).

\section{References}

Benick, G. \& Lohse, G.A. (1974) 14. Tribus: Callicerini (Athetae). In: Freude, H., Harde, K.W. \& Lohse, G.A. (Eds.), Die Käfer Mitteleuropas. Band 5, Staphylinidae II (Hypocyphtinae und Aleocharinae). Pselaphidae. Krefeld: Goecke \& Evers Verlag, pp. 72-220.

Bernhauer, M. (1899) Sechste Folge neuer Staphyliniden aus Europa, nebst Bemerkungen. Verhandlungen der $k$. $k$. zoologisch-botanischen Gesellschaft in Wien, 49, 422-437.

Bernhauer, M. (1906) Neue Aleocharinen aus Nordamerika. (II. Teil). Deutsche Entomologische Zeitschrift, 1906, Heft II, 337-348.

Bernhauer, M. (1907) Neue Aleocharini aus Nordamerika. (Col.) (3. Stück). Deutsche Entomologische Zeitschrift, 1907, 381-405.

Bernhauer, M. (1909) Neue Aleocharini aus Nordamerika. (Col.) (4. Stück.) Deutsche Entomologische Zeitschrift, 1909, Heft IV, 515-528.

Bernhauer, M. \& Scheerpeltz, O. (1926) Staphylinidae VI. In: Junk, W. \& Schenkling, S. (Eds.) Coleopterorum Catalogus, Pars 82. W. Junk, Berlin, 499-988.

Blackwelder, R. E. (1952) The generic names of the beetle family Staphylinidae, with an essay on genotypy. United States National Museum Bulletin, 200, 1-483.

Blatchley, W.S. (1910) An Illustrated Descriptive Catalogue of the Coleoptera or Beetles (exclusive of the Rhynchophora) Known to Occur in Indiana. Indianapolis: The Nature Publishing Co.: $1358 \mathrm{pp}$.

Casey, T. L. (1906) Observations of the staphylinid groups Aleocharinae and Xantholinini, chiefly of America. Transactions of the Academy of Sciences of St. Louis, 16(6), 125-434.

Casey, T. L. (1910a) New Species of the Staphylinid Tribe Myrmedoniini. Memoirs on the Coleoptera I. Lancaster: The New Era Printing Company, pp.1-183.

Casey, T. L. (1910b) Synonymical and other notes on Coleoptera. The Canadian Entomologist, 42(4), 105-114.

Fairmaire, L. (1883) Descriptions de Coléoptères recueillis par M. le Baron Bonnaire en Algérie. Bulletin et Annales de la Société Entomologique de Belgique, 27(30), xli-xlviii.

Fenyes, A. (1907) The Aleocharinae by Casey. Entomological News, 18(2), 60-61.

Fenyes, A. (1908) A preliminary systematic arrangement of the Aleocharinae (Coleoptera) of the United States and Canada. Entomological News, 19(2), 56-65.

Fenyes, A. (1920) Coleoptera. Fam. Staphylinidae, subfam. Aleocharinae. In: Wytsman, P. (Ed.), Genera Insectorum, Fasc. 173 B. Bruxelles: L. Desmet-Verteneuil, pp. 111-414. 
Fenyes, A. (1921) New genera and species of Aleocharinae with a polytomic synopsis of the tribes. Bulletin of the Museum of Comparative Zoology, 65, 17-36.

Gusarov, V.I. (2002) A revision of Nearctic species of the genus Geostiba Thomson, 1858 (Coleoptera: Staphylinidae: Aleocharinae). Zootaxa, 81, 1-88.

ICZN (1999) International Code of Zoological Nomenclature. Fourth Edition. London: The International Trust for Zoological Nomenclature. xxix $+306 \mathrm{pp}$.

Kiesenwetter, H. (1850) Funfzig Diagnosen unbeschriebener oder wenig bekannter Europäischer Käfer. Entomologische Zeitung, 11(7), 217-225.

Kraatz, G. (1857) Beiträge zur Kenntniss der Termitophilen. Linnaea Entomologica, 11, 44-56.

Muslant, E. \& Rey, C. (1874a) Tribu des Brévipennes. Famille des Aléochariens. Septième branche: Myrmédoniaires. Annales de la Société d'Agriculture, Histoire Naturelle et Arts utiles de Lyon, série 4. 6 [1873], 33-738.

Muslant, E. \& Rey, C. (1874b) Histoire Naturelle des Coléoptères de France. Brévipennes. Aléochariens. Suite. Paris: Deyrolle: vi+695 pp., pl. 1-5.

Newton, A.F., Thayer, M.K., Ashe, J.S. \& Chandler, D.S. (2000) Staphylinidae Latreille, 1802. In: Arnett, R.H., Thomas, M.C. (Eds.), American Beetles. Vol.1. Archostemata, Myxophaga, Adephaga, Polyphaga: Staphyliniformia. Boca Raton: CRC Press, pp. 272-418.

Pace, R. (1983) Il genere Tropimenelytron Scheerpeltz (Coleoptera, Staphylinidae). Nouvelle Revue d'Entomologie, 13(2), 185-190.

Sawada, K. (1970) Aleocharinae (Staphylinidae, Coleoptera) of the IBP-Station in the Shiga Heights, Central Japan (I). Bulletin of the National Science Museum, 13(1), 23-64.

Sawada, K. (1972) Methodological Research in the Taxonomy of Aleocharinae. Contributions from the Biological Laboratory, Kyoto University, 24(1), 31-59.

Seevers, C.H. (1978) A generic and tribal revision of the North American Aleocharinae (Coleoptera: Staphylinidae). Fieldiana: Zoology, 71, vi + 275 pp.

Thomson, C.G. (1858) Försök till uppställning af Sveriges Staphyliner. Öfversigt af Kongl. Vetenskaps-Akademiens Förkhandlingar, 15, 27-40.

Thomson C.G. (1867) Skandinaviens Coleoptera, synoptiskt bearbetade, Tom 9. Lund: Lundbergska Boktryckeriet: 408 pp. 